\title{
Mode of interaction of calcium oxalate crystal with human phosphate cytidylyltransferase 1: a novel inhibitor purified from human renal stone matrix
}

\author{
Priyadarshini Pathak ${ }^{1}$, Pradeep Kumar Naik ${ }^{1}$, Dipankar Sengupta ${ }^{1}$, Shrawan Kumar Singh ${ }^{2}$, \\ Chanderdeep Tandon ${ }^{1 *}$
}

${ }^{1}$ Biotechnology \& Bioinformatics, Jaypee University of Information Technology, Waknaghat, H.P., India;

${ }^{2}$ Department of Urology, Post Graduate Institute of Medical Education and Research (PGIMER), Chandigarh, India.

Email: ${ }^{*}$ chanderdeep.tandon@juit.ac.in; tandonchanderdeep@yahoo.com

Received 17 June 2011; revised 12 July 2011; accepted 10 August 2011.

\begin{abstract}
Nephrolithiasis is a common clinical disorder, and calcium oxalate $\left(\mathrm{CaO}_{x}\right)$ is the principal crystalline component in approximately $75 \%$ of all renal stones. It is widely believed that proteins act as inhibitors of crystal growth and aggregation. Acidic amino acids present in these proteins play a significant role in the inhibition process. In this study, interaction of calcium oxalate with human phosphate cytidylyltransferase 1(CCT), a novel calcium oxalate crystal growth inhibitor purified from human renal stone matrix has been elucidated in silico and involvement of acidic amino acids in the same. As only sequence of CCT is available, henceforth its 3-D structure was modeled via Homology modeling using Prime module of Schrodinger package. Molecular dynamic simulation of modeled protein with solvation was done by macromodel (Schrodinger). The quality of modeled protein was validated by JCSG protein structure validation (PROCHECK \& ERRAT) server. To analyze the interaction of modeled protein CCT with calcium oxalate along with role played by acidic amino acids, 'Docking simulation' was done using MOE-Dock. Interaction between calcium oxalate and CCT was also studied by substituting acidic amino acid in the active sites of the protein with neutral and positively charged amino acids. The in silico analysis showed the bond formation between the acidic amino acids and calcium atom, which was further substantiated when substitution of these acidic amino acids with alanine, glycine, lysine, arginine and histidine completely diminished the interaction with calcium oxalate.
\end{abstract}

Keywords: Calcium Oxalate; Matrix-Assisted Laser Desorption/ Ionization-Time of Flight
(MALDI-TOF-MS); Antilithiatic; Human Phosphate Cytidylyltransferase 1; MOE-Dock.

\section{INTRODUCTION}

Calcium oxalate is the most significant component of urinary stones [1]. The mechanisms for the formation of calcium oxalate urinary stones are still not understood, though it is thought that organic macromolecules, in particular proteins, play a significant role. It is widely believed that proteins act as inhibitors of crystal growth and aggregation. Prothrombin fragment 1 (PFT1) [2], nephrocalcin [3], bikunin [4,5] and osteopontin [6] have all been shown to inhibit growth or aggregation or both in crystallization experiments. Nucleolin-related protein (NRP) is found on the surface of inner medullary collecting duct (IMCD) cells in culture (cIMCD) and selectively adsorbs to calcium oxalate ( $\mathrm{CaOx})$. NRP mediates attachment to the renal tubular epithelium of Ca stone crystals through an electrostatic interaction with a highly acidic region (acidic fragment (AF)) similar to those of other proteins that have been reported to affect urinary crystal formation. In vitro studies have shown that OPN is a potent inhibitor of COM growth, promotes the formation of calcium oxalate dihydrate (COD) rather than monohydrate, and inhibits the aggregation of COM crystals [7].

In vivo, OPN is the major component of the organic matrix of oxalate-containing kidney stones. OPN contains; 300 amino acids, including a conserved sequence of contiguous aspartic acid residues [8]. This polypeptide chain undergoes extensive posttranslational modification, including glycosylation, phos-phorylation, and sulfation. The exact pattern of modification depends upon the species and tissue in which the protein is synthesized. The bovine milk isoform of OPN contains 27 
sites of serine phosphorylation, 1 of threonine phosphorylation, and 3 of O-linked glycosylation [9]. Human milk OPN is phos-phorylated at 34 serine and 2 threonine residues, and O-glycosylated at five sites [10].

The specific mechanism by which these proteins might interact with calcium oxalate surfaces is still in its infancy. Researchers working in other areas of biomineralization [11] have suggested that acidic amino acid residues such as Asp and Glu, that are expected to be deprotonated and negatively charged at urinary $\mathrm{pH}$, are attracted to the positively charged calcium ions. It would therefore be expected that proteins rich in $\gamma$-carboxyglutamic acid, Gla, such as PFT1, with two deprotonated carboxylate groups would be even better at electrostatically binding to calcium sites [12,13].

Very recently, we have isolated and characterized a novel protein, human phosphate cytidylyltransferase 1 , choline,beta (CCT) which is anionic in nature (MW 42 $\mathrm{kDa}$ ) from organic matrix of calcium oxalate renal stones, which inhibits the growth of calcium oxalate [14]. It was identified by MALDI-TOF-MS followed by database search on MASCOT server as human phos-phate cytidylyltransferase 1 , beta. Molecular weight of this novel $\mathrm{CaOx}$ crystal growth inhibitor from human renal stone matrix is also same as that of human phosphate cytidylyltransferase 1 , choline, beta. It is involved in the biosynthesis of phosphatidylcholine which happens to be an important constituent of human renal stones and is also reported to have an antilithiatic effect. Amino acid sequence of the identified protein revealed, presence of acidic amino acid. The aim of the present work is to study the interaction of this novel $\mathrm{CaO}_{x}$ crystal growth inhibitor (CCT) from human renal stone matrix with calcium oxalate at molecular level in silico.

\section{METHODS}

\subsection{Sequence Alignments, Secondary Structure Prediction and Protein Fold Recognition}

Blast search of the human CCT sequence obtained from MALDI-TOF-MS and MASCOT was done using nonredundant Protein Data Bank for sequence alignment and further selection of appropriate template for secondary structure prediction and fold recognition.

\subsection{Homology Modeling of Human CCT 1 Choline Beta}

Homology modeling of the identified human phosphate cytidylyltransferase 1 , choline, beta protein structure was done by using Prime module of Schrodinger (Prime version 1.5, Macromodel version 9.1, Schrodinger, LLC, New York, NY, 2005) software. Considering the high degree of similarity of crystal structure of mammalian CTP: phosphocholine cytidylyltransferase (3HL4_A) of
Rattus novergicus with CCT, it was taken as a model cytidylyltransferase (template) for studying structurefunction relationships $[15,16]$.

The structure of protein was modeled on the basis of its structural similarity with the chain A, crystal structure of a mammalian CTP: phosphocholine cytidylyltransferase (Protein Data Bank ID: 3HL4) of Rattus novergicus as a template. The degree of identity between the template and the human CCT sequence was 53\%, which enabled a preliminary model to be generated by Schrödinger. The sequence alignment was then improved manually and comparative homology method was used to build the structure of CCT. Macromodel program of Schrödinger software was used for molecular dynamic simulation. To check the quality of the modeled protein, JCSG protein structure validation server was used where PROCHEK \& ERRAT validation were done.

\subsection{Docking of Homology Model of Human CCT1 Choline Beta with Calcium Oxalate}

Calcium oxalate structure was drawn with the help of molecular builder of Molecular Operating Environment (MOE) package developed by the Chemical Computing Group Inc. Montreal, Canada. Active site of the modeled protein (CCT) was predicted by using active site finder tool of MOE software. Then, the docking of modeled protein with calcium oxalate was done using MOEDock. MOE-dock utilizes a Monte Carlo Simulated Annealing (SA) method in docking calculations to search for favorable binding configurations of a small, flexible ligand and a rigid macromolecule in a pre-set box. The docking energy calculation was carried out within a userspecified three-dimensional docking box (3D docking box) using the simulated annealing method under the OPLS-AA force field. The energy grids for docking were generated as grid-based potential fields by the MOEDock program, to reduce the calculation time. Each docking energy value was calculated as the sum value of the electrostatic, Van der Waals, and flexibility energies. The interaction energy was calculated using the electrostatic and Van der Waals potential fields sampled on a grid overlaying the 3D docking box. The 3D docking box was interpolated at the atom positions by tri-linear interpolation. The Van der Waals parameters were taken from the currently active force field. The electrostatic field was calculated based on forcefield in the Coulombic manner using the constant dielectric of 1.0 for solvation. MOE-Dock performed 25 independent docking runs, and wrote the resulting conformations and their energies to a molecular database file. The lowest docking energy conformation for each active site was chosen for LIGPLOT. 


\subsection{Point Mutation of Acidic Amino Acid in the Active Site of 1 and 2 of the Wild Type Protein WITH Neutral and Positively Charged Amino Acids}

To investigate the significance of acidic amino acids present in the active sites of the wild type modeled protein human CCT 1 choline beta, they were substituted with alanine, glycine, lysine, arginine and histidine in active sites 1 and 2. After incorporating these mutations, protein was docked with calcium oxalate using MOEDock with same parameter of docking as was used for wild type.

\section{RESULTS}

Before you begin to format your paper, first write and save the content as a separate text file. Keep your text and graphic files separate until after the text has been formatted and styled. Do not use hard tabs, and limit use of hard returns to only one return at the end of a paragraph. Do not add any kind of pagination anywhere in the paper. Do not number text heads-the template will do that for you.

Finally, complete content and organizational editing before formatting. Please take note of the following items when proofreading spelling and grammar:

\subsection{Sequence Alignments, Secondary Structure Prediction and Protein Fold Recognition}

The amino acid sequence of human CCT was 53\% similar (Figure 1(a)) to the chain A Ctp: phosphocholine cytidylyltransferase (Protein Data Bank ID: 3HL4) of Rattus novergicus.

\subsection{Homology Modeling of Human CCT1 Choline Beta and Docking with Calcium Oxalate}

The protein phosphate cytidylyltransferase 1 , choline, beta (CCT) was homology modeled based on sequence and structure align ments with the chain A Ctp: phosphocholine cytidylyltransferase (Protein Data Bank 3HL4_A) of Rattus novergicus (Figure 1(b)). After molecular simulation reliable structure was obtained (Figure 2). Two binding sites were predicted by the MOE site finder in the wild type CCT and were named site1 and site2. Calcium oxalate was docked within the active site using the Monte Carlo docking procedure of MOE. The best-ranking docking modes of the ligands were identified and energy minimized in the protein, while allowing full side chain flexibility. Docking of calcium oxalate with the two binding sites gave best docking score of $-31.07 \&-10.68$ with site1 \& site2 respectively (Table 1).

LIGPLOT analysis of the binding site reveals involvement of different amino acids of protein with calcium oxalate. In site1 (first binding site) Asp 82, Gly 83, Ile 84, His 168, Asp 169, Tyr 173 and Tyr 182 were involved in interaction with calcium oxalate (Figure 3(a)).In the site 2, Gln 54 and Tyr 107 were found to be participating in the interaction with calcium oxalate (Figure 3(b)). In the first binding site, most of the atoms-

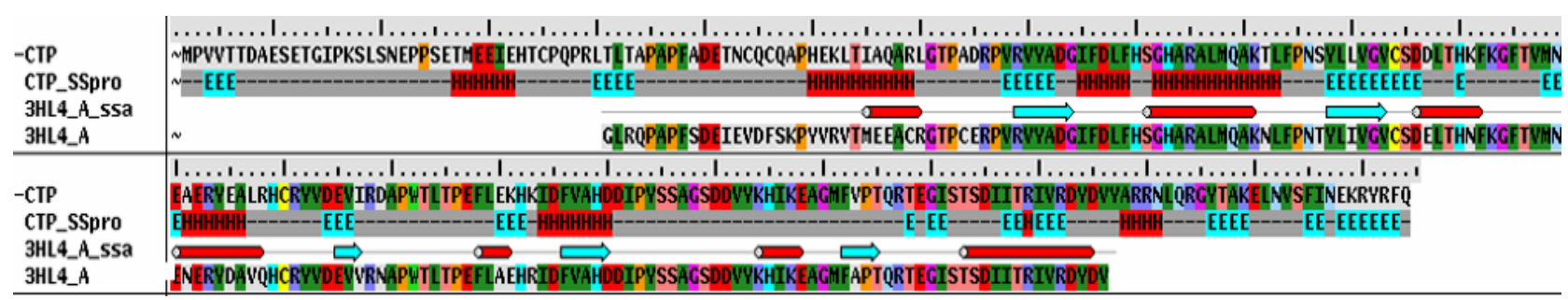

(a)

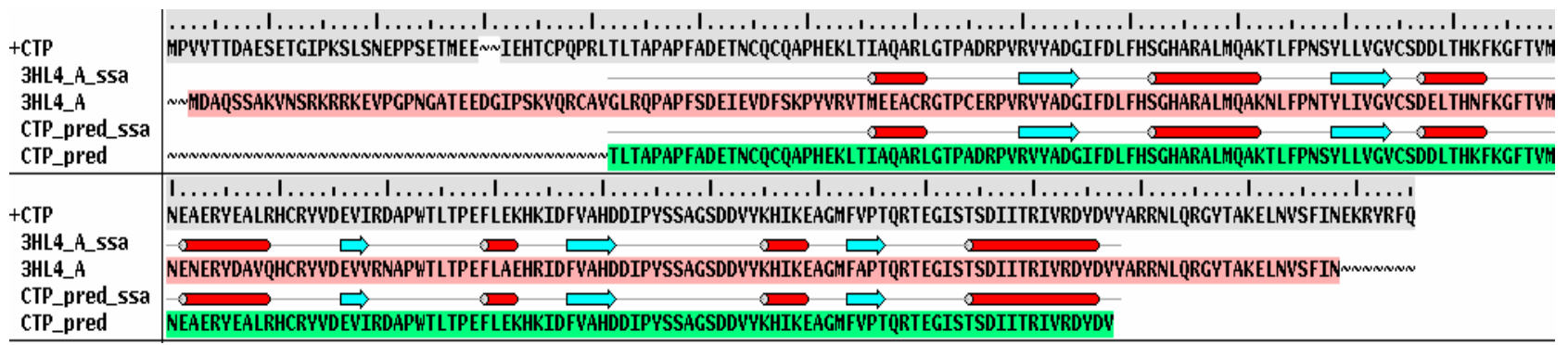

(b)

Figure 1. (a) Amino acid sequence analysis of human CCT and chain A Ctp: phosphocholine cytidylyltransferase (Protein Data Bank 3HL4_A) of Rattus novergicus. (b) Manual alignment of human CCT and chain A Ctp: phosphocholine cytidylyltransferase (Protein Data Bank 3HL4_A) of Rattus novergicus. 
Table1. Docking score of wild type human CCT model with calcium oxalate.

\begin{tabular}{|c|c|c|}
\hline $\begin{array}{l}\text { Wild Type } \\
\text { Human CCT }\end{array}$ & $\begin{array}{l}\text { Yite 1 } \\
\text { Y80, D82, G83, I84, F85, D86, H89, G91, } \\
\text { H92, R94, A95, Q98, C113, A149, P150, } \\
\text { W151, T152, L153, H168, D169, Y173, } \\
\text { S175, V181, Y182, T194, Q195, R196, T197, } \\
\text { E198, G199, I200, S201, T202, S203, D204, } \\
\text { R208 }\end{array}$ & $\begin{array}{l}\text { A48, T51, N52, } \frac{\text { Site } 2}{\text { C53, Q54, A57, P58, }} \\
\text { H59, E60, K61, L62, Q66, T71, A73, } \\
\text { D74, R75, P76, R78, Y107, L109, E144, } \\
\text { I146, K160, H161, L162, I163 }\end{array}$ \\
\hline Docking Score & -31.07 & -10.68 \\
\hline
\end{tabular}

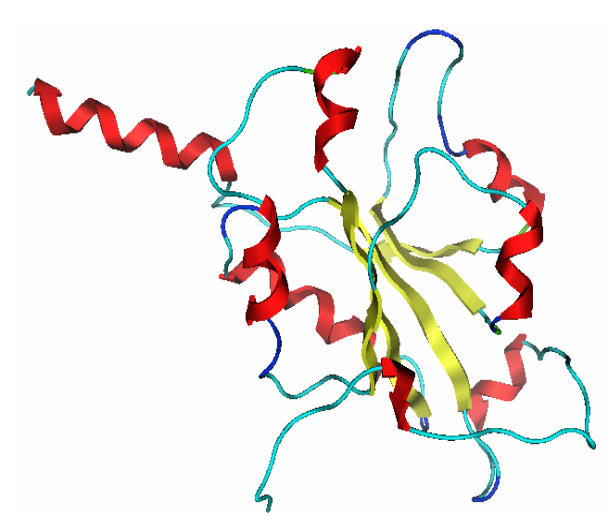

Figure 2. Homology modeled structure of CCT.

of calcium oxalate are highly exposed. Asp 82 and Tyr 182 are covalently bound to the calcium of the ligand (calcium oxalate), while Ile 84 is bound to the oxygen, where it is acting as side chain receptor (Figure 3(a)). In both of these active sites of the wild type modeled protein human CCT 1 choline beta, it was observed that acidic amino acids played a significant role in the interaction with calcium oxalate.

From Ramchandran plot, we found that for the CCT structure predicted by homology modeling $89.7 \%$ of residues have their torsion angles in the most favored regions, while remaining $10.3 \%$ have in additional allowed regions (Figure 4(a)). Further quality of modeled protein was checked using Errat program which revealed an overall quality factor $74.66 \%$ (Figure 4(b)) before and $80.14 \%$ (Figure 4(c)) after molecular simulation. These results indicate that quality of modeled protein is highly reliable.

\subsection{Point Mutation of Acidic Amino Acid of Active Site and Docking with Calcium Oxalate}

Upon substitution of acidic amino acids present in the active sites (site 1 and site 2) of the wild type modeled protein CCT with alanine, glycine, lysine, arginine and histidine, positive docking scores were obtained indicating a poor interaction with calcium oxalate. No docking score was found when acidic amino acid present in binding site1was mutated to arginine and histidine. In all of these active sites of the wild type modeled protein CCT, it was observed that acidic amino acids played a significant role in the interaction with calcium oxalate. These studies clearly demonstrate that acidic amino acids present in the wild type CCT showed a good docking score, an indicator of interaction with calcium an indicator of interaction with calcium oxalate, while substitution of these acidic amino acids with alanine, glycine, lysine, arginine and histidine gave a poor docking score (Table 2).

\section{DISCUSSION AND CONCLUSION}

Of all types of renal stones, calcium oxalate $\left(\mathrm{CaO}_{x}\right)$ is the most common composition found by chemical analysis [17]. $\mathrm{CaO}_{x}$ crystal growth inhibitors (proteins, lipids, glycosami-noglycans, and inorganic compounds) have been proposed to play an important role in renal stone disease $[18,19]$. During the last few years more and more research has been done at the cellular and molecular levels. In spite of these advances however, the clinical treatment of urolithiasis remain far from satisfactory. Stone recurrence in human beings cannot be predicted and is beyond the control of urologists, mainly because the mechanism of stone formation at molecular level is not yet fully understood [20]. Thus, determining the molecular mechanisms by which urinary constituents modulate calcium oxalate crystallization is crucial for understanding and controlling urolithiassis in humans.

Although a few initial molecular-scale investigations of the con-trolling mechanisms of kidney stone formation by these inhibitory molecules have been recently performed [21-24], the majority of previous studies have been concerned with the overall kinetics of crystallization, rather than molecular mechanisms which remain poorly defined. Therefore, in the present work, we have focused our attention on the interaction of the purified (in our lab) human phosphate cytidylyltransferase 1 (CCT) [14] with calcium oxalate using bioinformatics tools.

Cytidylyltransferases are critical enzymes involved in the biosyn-thetic pathways of lipids and complex carbohydrates. These enzymes catalyze a major step of energy input into biosynthesis by forming the activated intermediates, CDP-alcohols and CMP-sugars [25]. In fact CCT is involved in the biosynthesis of phosphatidylcholine which happens to be an important constituent of human renal stones and is also reported to have an anti- 
lithiatic effect.

Several cytidylyltransferases belong to a single family of structures, as defined by sequence similarities and signature sequence that occur in their catalytic domains. Mammalian CCT $\alpha$ contains several functional regions: an $\mathrm{N}$-terminal nuclear localization signal, a central catalytic domain, a membrane/lipid activation segment and a C-terminal phosphorylation region. There is a high degree of sequence similarity within the catalytic domain of all known forms of CCT, with the yeast catalytic domain being $56 \%$ identical to that of mammalian $\mathrm{CCT} \alpha$, and the catalytic domains of $\operatorname{CCT} \alpha$, and $\operatorname{CCT} \beta$ being $90 \%$ identical [16].

The degree of identity between the template and the human CCT sequence was 53\%, which enabled a preliminary model to be generated by Schrodinger. Aspartic acid, isoleucine, tyrosine and glycine were found to be interacting with calcium oxalate at site1. More negative the docking score, stronger is the binding between ligand and protein's active site [26]. The strong interaction between CCT's active site and calcium oxalate predicts inhibition of the same. Highest docking score (-31.079)

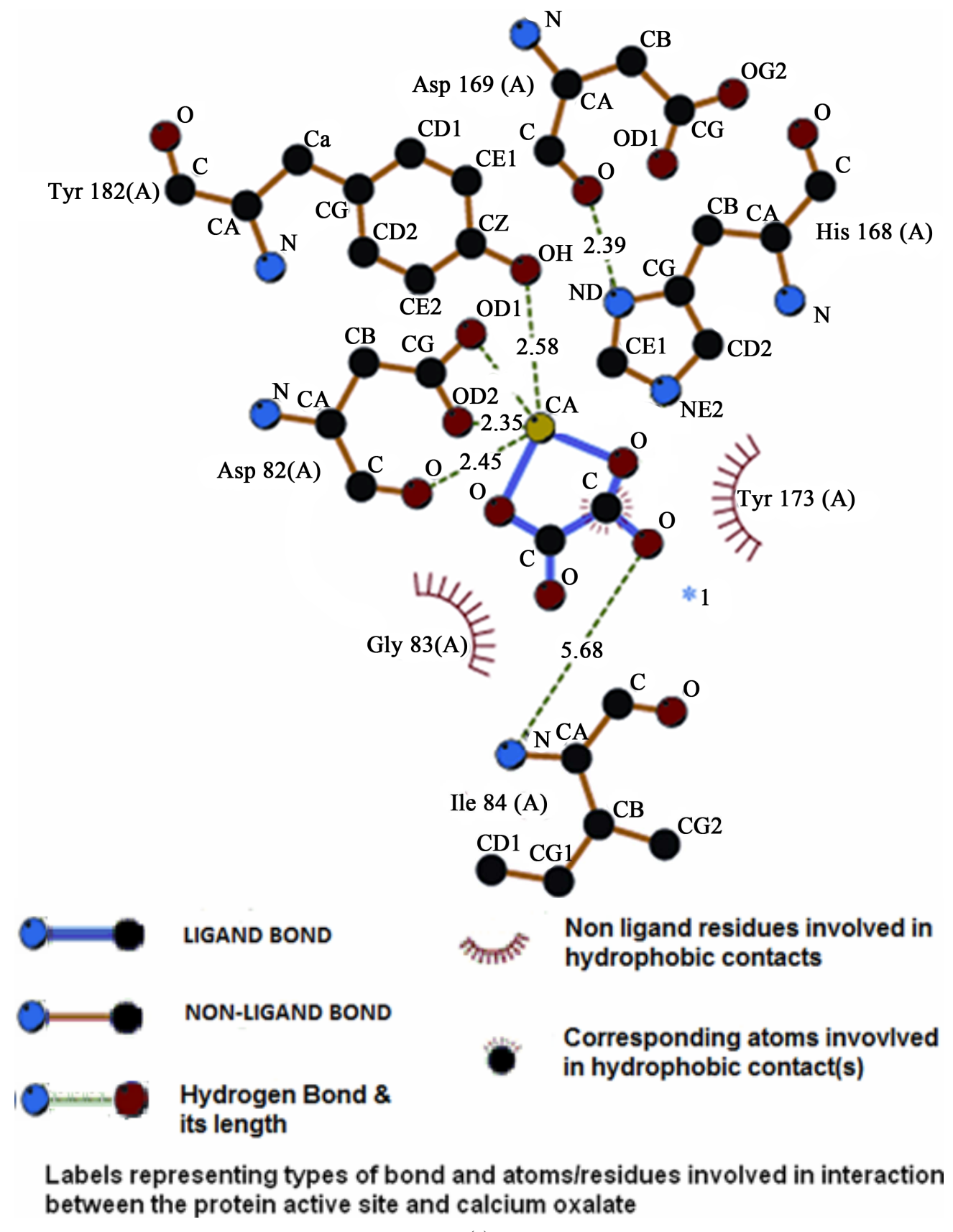

(a) 


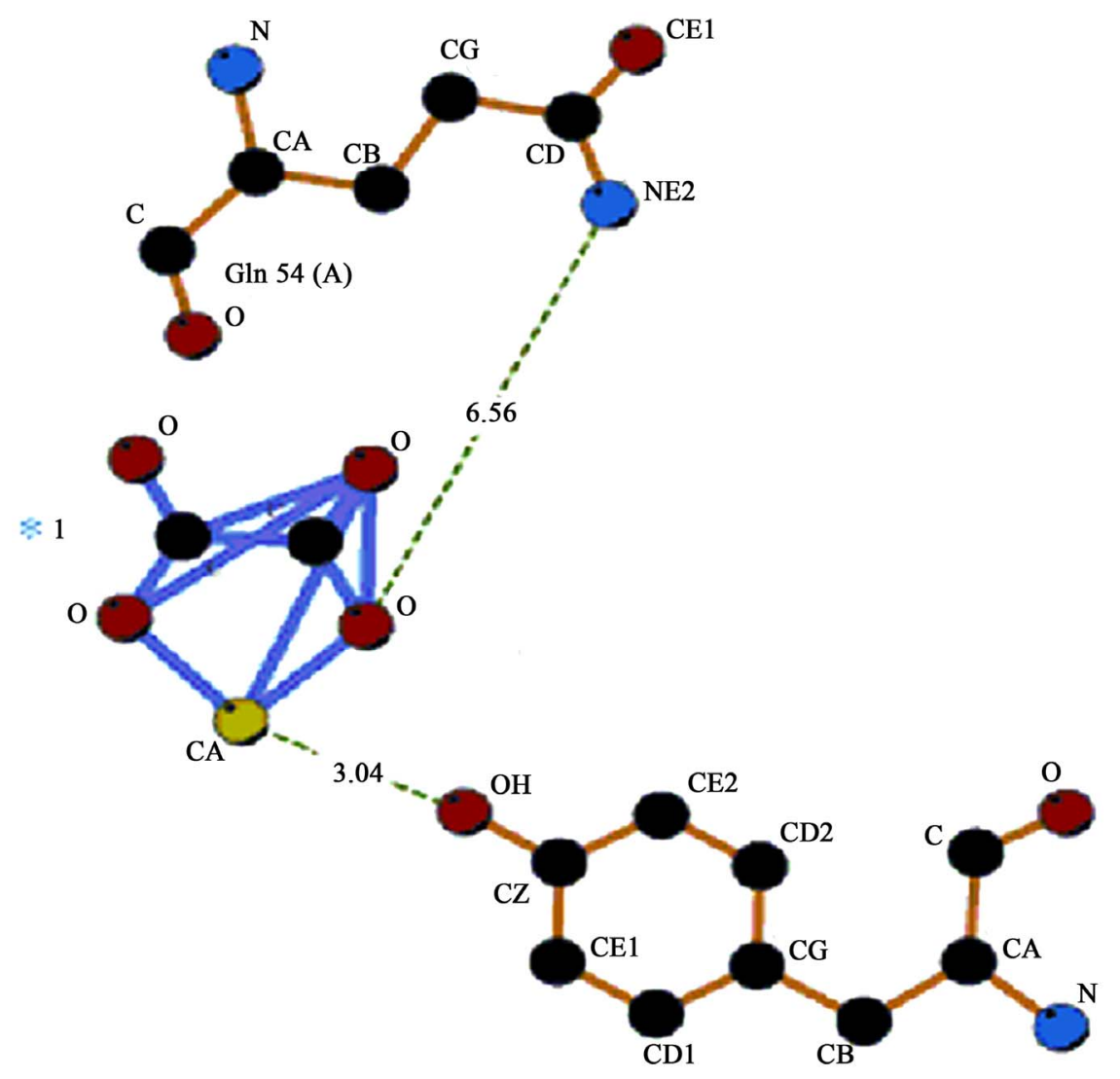

Tyr 107 (A)

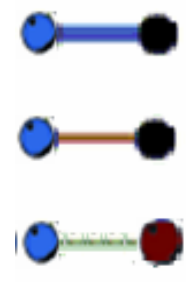

LIGAND BOND

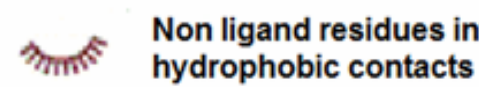

NON-LIGAND BOND

Hydrogen Bond \& its length

\section{Labels representing types of bond and atoms/residues involved in interaction between the protein active site and calcium oxalate}

(b)

Figure 3. (a) Asp 82, Gly 83, Ile 84, His 168, Asp 169, Tyr 173 and Tyr 182 were involved in interaction with calcium oxalate. (b) In the site 2, Gln 54 and Tyr 107 were found to be participating in the interaction with calcium oxalate.

with site1 indicates good binding of modeled protein with the ligand calcium oxalate. LIGPLOT of site1 showed involvement of aspartic acid (Asp) at position 82 with calcium while isoleucine (Ile) at position 84, interacts with oxygen of oxalate group. The binding site1 was found to be better for interaction with calcium oxalate compared to the other site. Whether a protein or other macromolecule acts as an inhibitor of growth and aggregation or a promoter of nucleation and aggregation implies that there must be some mechanism to explain the interaction with the mineral oxalate surfaces. The interaction between calcium and acidic Asp, Glu and Gla is certainly plausible, but it is equally conceivable that basic residues that are normally protonated at urinary $\mathrm{pH}$ and positively charged might experience an attraction toward negatively charged oxalate groups [27]. This is corroborated by the presence of basic amino acids too in the inhibitory proteins [28]. In either case steric constraints from 3D conformation of the molecule might limit the number of these simple interactions [27]. Positive docking score with mutated binding sites confirms the inhibitory role of acidic amino acids [29-31]. 


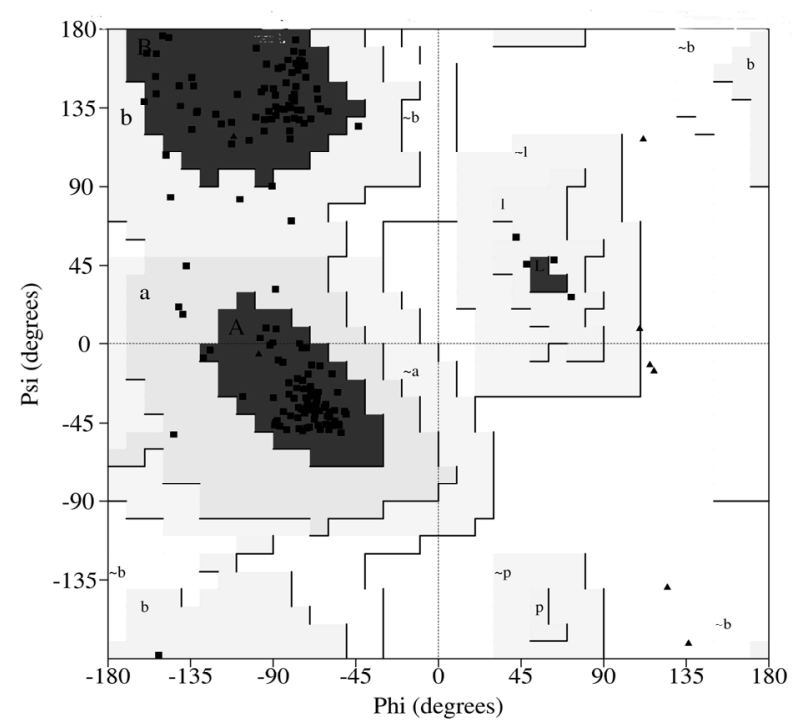

(a)



(b)

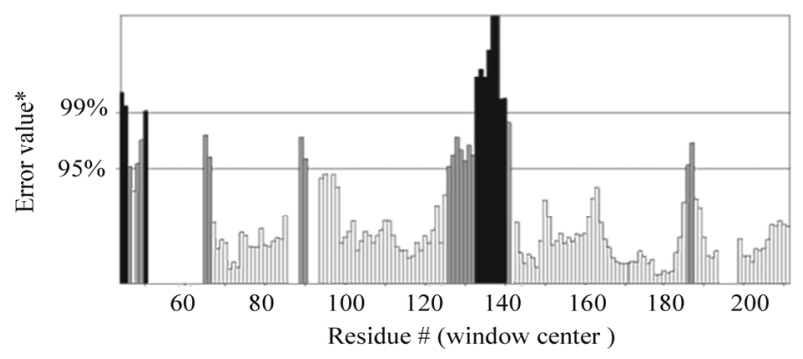

(c)

Figure 4. (a) In Ramchandran plot $89.7 \%$ of residues have their torsion angles in the most favored regions, while remaining $10.3 \%$ have in additional allowed regions. b \& c Errat program which revealed an overall quality factor $74.66 \%$ (b) before and $80.14 \%$ (c) after molecular simulation.

There are reports that members of cytidylyltransferase family have similar conserved sequence [32]. In our study, we found $100 \%$ sequence in binding site 1 and $29 \%$ sequence in site2, in the conserved region of CCT. Interestingly, conserved amino acids are contained within the catalytic core of the CCTs [32].

We found that the acidic amino acid is interacting with the calcium of calcium oxalate. This was further sub-
Table 2. Docking score of mutated human CCT modelwith calcium oxalate.

\begin{tabular}{lll}
\hline $\begin{array}{l}\text { Amino acids with } \\
\text { which point muta- } \\
\text { tion is done at spe- } \\
\text { cific positions in Site } \\
1 \text { \& } 2\end{array}$ & $\begin{array}{l}\text { Site 1 } \\
\text { Point mutation of } \\
\text { Aspartic acid at } \\
\text { position 82, 86, } \\
\text { 169, 204 \& Glu- } \\
\text { tamic acid at posi- } \\
\text { tion 198 }\end{array}$ & $\begin{array}{l}\text { Point mutation of } \\
\text { Aspartic acid at } \\
\text { position 74 \& } \\
\text { Glutamic acid at } \\
\text { position 60 \& 144 }\end{array}$ \\
\hline \multicolumn{1}{c}{ Alanine (A) } & 63.29 & 87.81 \\
Glycine (G) & 193.39 & 159.26 \\
Lysine (K) & 98.33 & 138.05 \\
Arginine (R) & 105.27 & 151.53 \\
Histidine (H) & 163.29 & 153.29 \\
\hline
\end{tabular}

stantiated when substitution of these acidic amino acids with alanine, glycine, lysine, arginine and histidine completely diminished the interaction with calcium oxalate. These findings are in conformity with the presence of acidic amino acids in the various inhibitors of calcification from human beings [29-31] as well as acidic nature of antilithiatic proteins from plants [33,34].

\section{FUNDING}

We thank Jaypee University of Information Technology, Solan, HP, India for providing funds to carry out this research work.

\section{ACKNOWLEDGEMENTS}

We express our gratitude to the Department of Urology, Post Graduate Institute of Medical Education and Research (PGIMER) Chandigarh, India for providing kidney stones. We would also like to thank Mr. Deeptak Verma for helping us with MOE simulation studies at University of North Carolina, Charlotte.

\section{REFERENCES}

[1] Drach, G.W. (1992) Urinary lithiasis etiology, diagnosis, and medical management. Campbell's Textbook of Urology, 2085.

[2] Grover, P.K. and Ryall, R.L. (1998) Inhibition of calcium oxalate crystal growth and aggregation by prothrombin and its fragments in vitro. European Journal of Biology Chemistry, 263, 50.

[3] Nakagawa, Y., Abram, V., Kezdy, F.J., Kaiser, E.T. and Coe, F.L. (1983) Purification and characterization of the principal inhibitor of calcium oxalate monohydrate crystal growth in human urine. The Journal of Biological Chemistry, 258, 12594-12600.

[4] Atmani, F. and Khan, S.R. (1995) Characterization of uronic acid rich inhibitor of calcium oxalate crystallization isolated from rat urine. Urological Research, 23, 95.

[5] Atmani, F., Mizon, J. and Khan, S. R. (1996) Identification of uronic acid rich protein as urinary bikunin, the light chain of inter-a-inhibitor. European Journal of Biology Chemistry, 236, 984.

[6] Wesson, J.A., Johnson, R.J. and Mazzali, M.A, et al. (2003) Osteopontin is a critical inhibitor of calcium ox- 
alate crystal formation and retention in renal tubules. Journal of the American Society Nephrology, 14, 139147.

[7] Taller, A., Grohe, B., Rogers, K.A., Goldberg, H.A. and Hunter, G.K. (2007) Specific adsorption of osteopontin and synthetic polypeptides to calcium oxalate monohydrate crystals. Biophysical Journal, 93, 1768-1777.

[8] Sodek, J., Ganss, B. and McKee, M.D. (2000) Osteopontin. Critical Reviews in Oral Biology and Medicine, 11, 279-303.

[9] Sorensen, E.S., Hojrup, P. and Petersen, T.E. (1995) Posttranslational modifications of bovine osteopontin: identification of twenty-eight phosphorylation and three O-glycosylation sites. Protein Scienty, 4, 2040-2049.

[10] Christensen, B., Nielsen, M.S., Haselmann, K.F., Petersen, T.E. and Sorensen, E.S. (2005) Posttranslationally modified residues of native human osteopontin are located in clusters: identification of 36 phosphorylation and five O-glycosylation sites and their biological implications. Biochemical Journal, 390, 285-292.

[11] Addadi, L. and Weiner, S. (1985) Interactions between acidic proteins and crystals: Stereochemical requirements in biomineralization. Proceedingof the Natlional Academy Science of the USA, 82, 4110.

[12] Lian, J.B., Prien, E.L., Glimcher, M.J. and Gallop, P.M. (1977) The presence of protein bound ccarboxyglutamic acid in calcium-containing renal calculi. Journal of Clinicine Investigation, 59, 1151.

[13] Ryall, R.L., Fleming, D.E. and Grover, P.K. et al. (2000) The hole truth: Intracrystalline proteins and calcium oxalate kidney stones. Molecular Urology, 4, 391.

[14] Priyadarshini, Singh, S.K. and Tandon, C. (2009) Mass spectrometric identification of human phosphate cytidylyltransferase 1 as a novel calcium oxalate crystal growth inhibitor purified from human renal stone matrix. Clinica Chimica Acta, 408, 34-38.

[15] Lee, J., Johnson, J., Ding, Z., Paetzel, M. and Cornell, R.B. (2009) Crystal structure of a mammalian CTP: phosphocholine cytidylyltransferase catalytic domain reveals novel active site residues within a highly conserved nucleotidyltransferase fold. The Journal of Biological Chemistry, 284, 33535-33548.

[16] Jon, A.F., Heidi, A.C. and Claudia Kent. (1999) Enzymatic and cellular characterization of a catalytic fragment of CTP: Phosphocholine Cytidylyltransferase $\alpha$. The Journal of Biological Chemistry, 274, 13384-13389.

[17] Coe, F.L., Parks, J.H. and Asplin, J.R. (1992) The pathogenesis and treatment of kidney stones. New England Journal of Medicine, 327, 1141-1152. doi:10.1056/NEJM199210153271607

[18] Zerwekh, J.E., Holt, K. and Pak, C.Y. (1983) Natural urinary macromolecular inhibitors: attenuation of inhibitory activity by urate salts. Kidney International, 23, 838-841.

[19] Coe, F.L., Parks, J.H. and Nakagawa, Y. (1991) Protein inhibitors of crystallization. Seminars in Nephrology, 11, 98-109.

[20] Aggarwal, S., Tandon, C., Forouzandeh, M., Singla, S.K., Kiran, R. and Jethi, R.K. (2000) Role of biomolecules from human renal stone matrix on COM crystal growth. Molecular and Cellular Biochemistry, 210, 109-119.

[21] Shirane, Y., Kurokawa, Y., Miyashita, S., Komatsu, H. and Kagawa, S. (1999) Study of inhibition mechanisms of glycosaminoglycans on calcium oxalate monohydrate crystals byatomic force microscopy. Urology Research, 27, 426-431.

[22] Guo, S., Ward, M.D. and Wesson, J.A. (2002) Direct visualization of calcium oxalate monohydrate (COM) crystallization and dissolution with atomic force microscopy (AFM) and the role of polymeric additives. Langmuir, 18, 4284-4291.

[23] Qiu, S.R., Wierzbicki, A. and Orme, C.A. et al. (2004) Molecular modulation of calcium oxalate crystallization by osteopontin and citrate. Proceedingof the Natlional Academy Science of the USA, 101, 1811-1815.

[24] Jung, T., Sheng, X., Choi, C.K., Kim, W., Wesson, J.A. and Ward, M.D. (2004) Probing crystallization of calcium oxalate monohydrate and the role of macromolecule additives with in situ atomic force microscopy. Langmuir, 20, 8587-8596.

[25] Weber, C.H., Park, Y.S., Sanker, S., Kent, C. and Ludwig, M.L. (1999) A prototypical cytidylyltransferase: CTP: Glycerol-3 phosphate cytidylyltrnsferase from Bacillus subtilis. Structure 7, 9.

[26] Bijarnia, R.K., Kaur, T., Naik, P.K., Singla, S.K. and Tandon C. (2008) In silico study on interaction of active binding sites of proteins with calcium oxalate monohydrate. Office of James Bumett, 2, 92-107.

[27] Gul, A. and Rez, P. (2007) Models for protein binding to calcium oxalate surfaces. Urology Research, 35, 63-71.

[28] Tandon, C., Aggarwal, S., Forouzandeh, M. and Jethi, R.K. (1998) Inhibitors of in vitro mineralization from rabbit aorta and their role in biomineralization. Journal of Cellular Biochemistry, 68, 287-297.

[29] Nakagawa, Y., Ahmed, M., Hall, S.L., Deganello, S. and Coe F.L. (1987) Isolation from human calcium oxalate renal stones of nephrocalcin, a glycoprotein inhibitor of calcium oxalate crystal growth. Evidence that nephrocalcin from patients with calcium oxalate nephr- olithiasis is deficient in $\gamma$-carboxyglutamic acid. Journal of Clinical Investigation, 79, 1782-1787.

[30] Nakagawa, Y., Abram, V., Parks, J.H., Lau, H.S.H., Kawooya, J.K. and Coe, F.L. (1985) Urine glycoprotein crystal growth inhibitors. Evidence for a molecular abnormality in calcium oxalate nephrolithiasis. Journal of Clinical Investigation, 76, 1455-1462.

[31] Shiraga, H., Min, W., VanDusen, W.J. et al. (1992) Inhibition of calcium oxalate crystal growth in vitro by uropontin: Another member of the aspartic acid rich protein superfamily. Proceedingof the Natlional Academy Science of the USA, 89, 426-430.

[32] Kalmar, G.B., Kay, R.J., Lachance, A., Aebersold, R. and Cornell, R.B. (1990) Cloning and expression of rat liver CTP: Phosphocholine cytidylyltransferase: an amphipathic protein that controls phosphatidylcholine synthesis. Proceedingof the Natlional Academy Science of the USA, 87, 6029-6033.

[33] Kaur, T., Bijarnia, R.K., Singla, S.K. and Tandon, C. (2009) Purification and characterization of an anticalcifying protein from the seeds of trachyspermum ammi (L.). Protein and Peptides Letters, 16, 173-81.

[34] Bijarnia, R.K., Kaur, T., Singla, S.K. and Tandon, C. (2009) A novel calcium oxalate crystal growth inhibitory protein from the seeds of Dolichos biflorus (L.). The Protein Journal, 28, 161-168. 